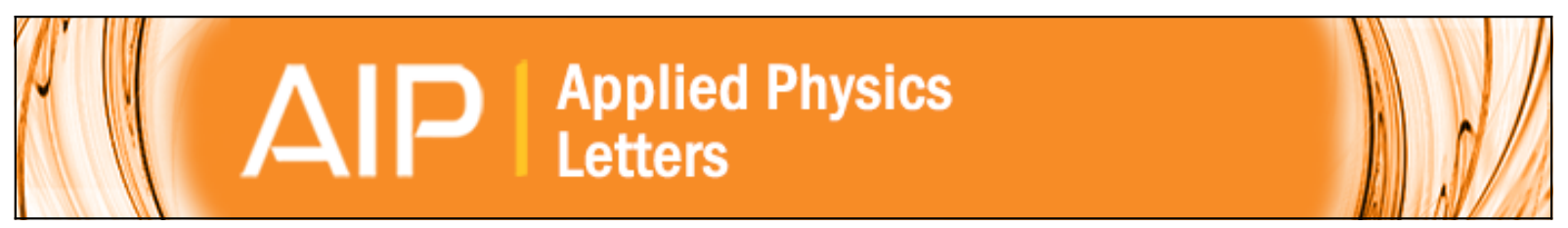

\title{
Boiling on spatially controlled heterogeneous surfaces: Wettability patterns on microstructures
}

HangJin Jo, Dong In Yu, Hyunwoo Noh, Hyun Sun Park, and Moo Hwan Kim

Citation: Applied Physics Letters 106, 181602 (2015); doi: 10.1063/1.4919916

View online: http://dx.doi.org/10.1063/1.4919916

View Table of Contents: http://scitation.aip.org/content/aip/journal/apl/106/18?ver=pdfcov

Published by the AIP Publishing

\section{Articles you may be interested in}

Reversed boiling curve phenomenon on surfaces with interlaced wettability

AIP Advances 4, 107110 (2014); 10.1063/1.4897953

Separate effects of surface roughness, wettability, and porosity on the boiling critical heat flux

Appl. Phys. Lett. 103, 024102 (2013); 10.1063/1.4813450

Fast photo-switchable surfaces for boiling heat transfer applications

Appl. Phys. Lett. 101, 191603 (2012); 10.1063/1.4766345

Do surfaces with mixed hydrophilic and hydrophobic areas enhance pool boiling?

Appl. Phys. Lett. 97, 141909 (2010); 10.1063/1.3485057

Effects of nanoparticle deposition on surface wettability influencing boiling heat transfer in nanofluids Appl. Phys. Lett. 89, 153107 (2006); 10.1063/1.2360892

LIVE DEMO

The Basics of COMSOL in 18 Minutes

จ๐ $\subset$ MSOL

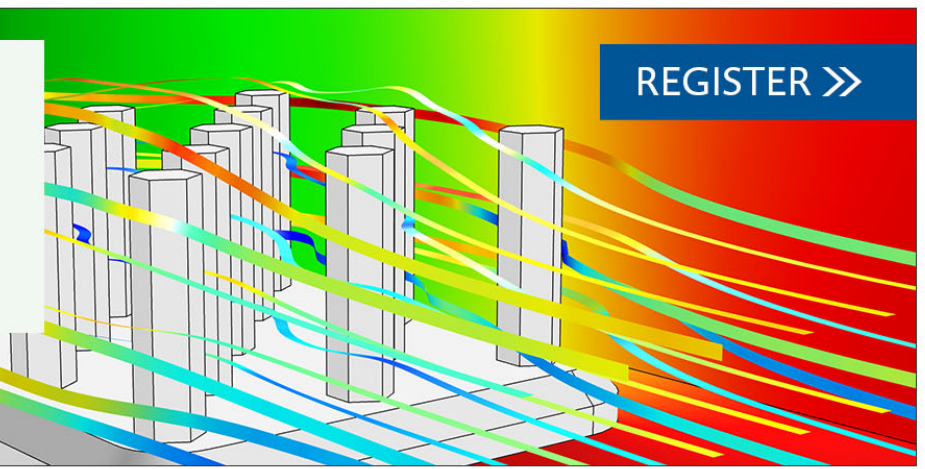




\title{
Boiling on spatially controlled heterogeneous surfaces: Wettability patterns on microstructures
}

\author{
HangJin Jo, ${ }^{1, a), b)}$ Dong In Yu, ${ }^{1, a)}$ Hyunwoo Noh, ${ }^{2}$ Hyun Sun Park, ${ }^{1, c)}$ \\ and Moo Hwan Kim ${ }^{1,2, c), d)}$ \\ ${ }^{1}$ Division of Advanced Nuclear Engineering, POSTECH, Two-Phase Flow Lab, San 31, HyojaDong, \\ Pohang 790-784, South Korea \\ ${ }^{2}$ Department of Mechanical Engineering, POSTECH, Two-Phase Flow Lab, San 31, HyojaDong, \\ Pohang 790-784, South Korea
}

(Received 20 February 2015; accepted 28 April 2015; published online 7 May 2015)

\begin{abstract}
We investigated nucleate boiling heat transfer with precisely controlled wetting patterns and micro-posts, to gain insights into the impact of surface heterogeneity. To create heterogeneous wetting patterns, self-assembled monolayers (SAMs) were spatially patterned. Even at a contact angle $<90^{\circ}$, bubble nucleation and bubble frequency were accelerated on SAM patterns, since this contact angle is larger than that found on plain surfaces. Micro-posts were also fabricated on the surface, which interrupted the expansion of generated bubbles. This surface structuring induced smaller bubbles and higher bubble frequency than the plain surface. The resistance provided by surface structures to bubble expansion broke the interface between the vapor mushroom and the heating surface, and water could therefore be continuously supplied through these spaces at high heat flux. To induce synergistic effects with wetting patterns and surface structures on boiling, we fabricated SAM patterns onto the heads of micro-posts. On this combined surface, bubble nucleation was induced from the head of the micro-posts, and bubble growth was influenced by both the SAM pattern and the micro-post structures. In particular, separation of the vapor path on the SAM patterns and the liquid path between micro-post structures resulted in high heat transfer performance without critical heat flux deterioration. ( 2015 AIP Publishing LLC.

[http://dx.doi.org/10.1063/1.4919916]
\end{abstract}

Improvement of nucleate boiling by modification of the heating surface has received significant attention for performance optimization of heat generating systems. ${ }^{1-4}$ In particular, modulation of surface wettability and structure has been investigated extensively. The wettability and surface structure of a heating surface affect bubble initiation, ${ }^{5-7}$ bubble dynamics, ${ }^{8,9}$ and nucleation site density. ${ }^{10}$ Mikic and Rohsenow ${ }^{11}$ developed a well-known boiling correlation by considering the effects of surface wettability and geometric constraints to derive a boiling heat transfer (BHT) coefficient. Surface characteristics also affect the critical heat flux (CHF), representing the limit of nucleate boiling; the CHF is enhanced by surface characteristics that influence force balance or liquid supplement. ${ }^{12-14}$

Recently, use of a heterogeneous wetting surface has been suggested for simultaneous improvement of BHT and CHF., ${ }^{75-17}$ Modified heating surfaces with mixed hydrophobic-hydrophilic wettability have exhibited BHT enhancements compared with homogeneous wetting surfaces. BHT enhancement on a heterogeneous wetting surface is attributed to hydrophobic patterns inducing bubble nucleation at lower superheat and continuous bubble generation. At $\theta>90^{\circ}$, when the bubble departs from the heating surface, part of the departing bubble remains and forms the next

\footnotetext{
${ }^{a)}$ H.J. Jo and D. I. Yu contributed equally to this work.

b) Dr. HangJin Jo is currently working in University of Wisconsin-Madison.

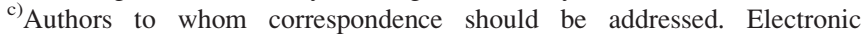
addresses: hejsunny@postech.ac.kr and mhkim@postech.ac.rk

${ }^{d)}$ Professor Moo Hwan Kim is currently working in Korea Institute of Nuclear Safety (KINS).
}

bubble, resulting in continuous bubble growth on the heating surface. ${ }^{7}$ However, continuous bubble generation on a hydrophobic pattern may block the supply of liquid and cause cooling along the pattern. Intuitively, therefore, certain hydrophobic patterns could cause BHT deterioration and CHF decrement at high heat flux; hence, the pattern should be designed to mitigate these effects. ${ }^{18}$ More recently, Betz et al. ${ }^{19}$ combined a nanostructured heating surface and patterned wetting to achieve the largest observed BHT on a mixed wetting surface with non-uniform spatial wettability. However, though the combination of wetting pattern and surface structures showed the large enhancement, the effects of wetting pattern and surface structure on boiling were not investigated separately and synergistic effects were not considered.

In this paper, we investigated nucleate boiling heat transfer with a precisely controlled microstructure and heterogeneous wetting pattern, to gain insights into the impact of surface heterogeneity on boiling. We suggest a combined surface, comprising a wetting pattern precisely located at the head of surface microstructures, to induce synergistic effects. In particular, a hydrophilic self-assembled monolayer (SAM) pattern fabricated onto the head of surface microstructures acted to separate vapor and liquid paths during vigorous bubble generation, and successive BHT improvements were observed without low CHF or BHT deterioration at high heat flux.

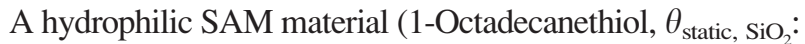
$57^{\circ}, \theta_{\text {static, }}$ SAM: $81^{\circ}$ ) was locally patterned onto the surface. As discussed above, even if BHT improvement is induced by a heterogeneous wetting surface, CHF decrement may result 
during interaction with hydrophobic patterns. ${ }^{18}$ In this study, however, by designing the pattern such that $\theta_{\mathrm{SAM}}<90^{\circ}$, vapor blocking by continuous bubble generation was prevented. The SAM pattern diameter was $100 \mu \mathrm{m}$ and the center-to-center pitch distance between patterns was $130 \mu \mathrm{m}$. Micro-post structures on the test sections were fabricated using photoresist lithography and a dry etching method. As shown in Fig. 1, the dimensions of the micro-post structures were measured using scanning electron microscopy (SEM) and a 3D-profiler. The micro-post diameter, micro-post height, and pitch distance between micro-posts were 110, 20, and $130 \mu \mathrm{m}$, respectively. The micro-post diameter was designed to be slightly larger than the SAM pattern diameter, such that the SAM pattern could be fabricated onto the top of a micro-post. A thin film metal layer was deposited on the reverse of the test samples for electrical connections and measurement of the wall temperature of the sample. A detailed description of the preparation method for the test samples is given in supplementary material. ${ }^{20}$ All pool boiling experiments were conducted after degassing for $2 \mathrm{~h}$ under atmospheric saturated condition, with distilled water.

Fig. 2 shows BHT enhancement on the heterogeneously modified surface. The SAM patterned surface maintained a lower superheat continuously across all heat fluxes, compared to a plain sample under same heat flux conditions. The difference in superheat between patterned and plain surfaces ranged from 3.1 to $4.9 \mathrm{~K}$, corresponding to BHT enhancements of $7 \%-24 \%$ for the patterned surface. This confirms that use of hydrophilic wetting patterns can result in BHT enhancement at high heat flux, without $\mathrm{CHF}$ decrease. The micro-post structured surface showed $23 \%$ BHT enhancement at $q^{\prime \prime}=730 \mathrm{~kW} / \mathrm{m}^{2}$ with a $114 \%$ improvement in CHF compared to the plain surface. The combined surface, including both wetting patterns and micro-post structures, showed excellent performance for both parameters: $45 \%-72 \%$ BHT enhancement in the nucleate boiling regime and $119 \%$ improvement in CHF compared with the plain surface. The simultaneous improvement of BHT and $\mathrm{CHF}$ on the combined surface is comparatively higher than boiling performance gains reported on other microstructured surfaces. ${ }^{20}$ A description of BHT enhancement on the modified surface requires consideration of nucleation conditions and nucleated bubble dynamics.

Because of the sub-nanometer roughness of the silicon wafer used as a substrate in this study, and the geometrical limit of the fabricated towering micro posts, not all heating surfaces used could be described by the vapor-trapping theory, ${ }^{21}$ since the heating surface was unable to trap vapor

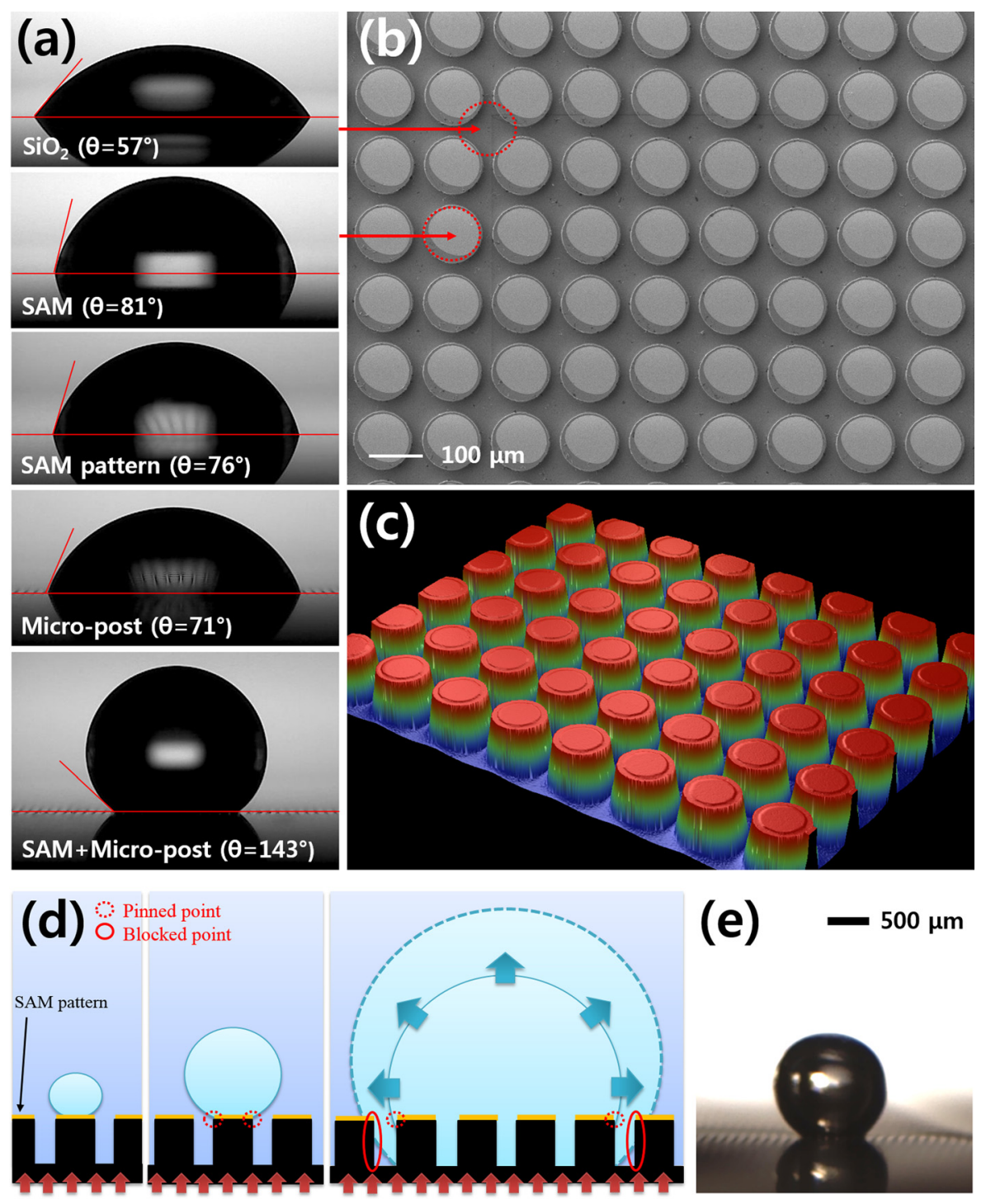

FIG. 1. Test sections (a) contact angles (plain $\left(\mathrm{SiO}_{2}\right)$ surface, SAM-coated surface, SAM-patterned surface, micropost structured surface, and micro-post structured surface with SAM patterns), (b) SEM image of micro-post structured surface with SAM patterns, (c) 3D-profile image of micro-post structured surface with SAM patterns, (d) a rendering of the model of the bubble nucleation and growth on the micropost structured surface with SAM patterns, and (e) single bubble image on the SAM+Micro-post surface in boiling. 
(a)

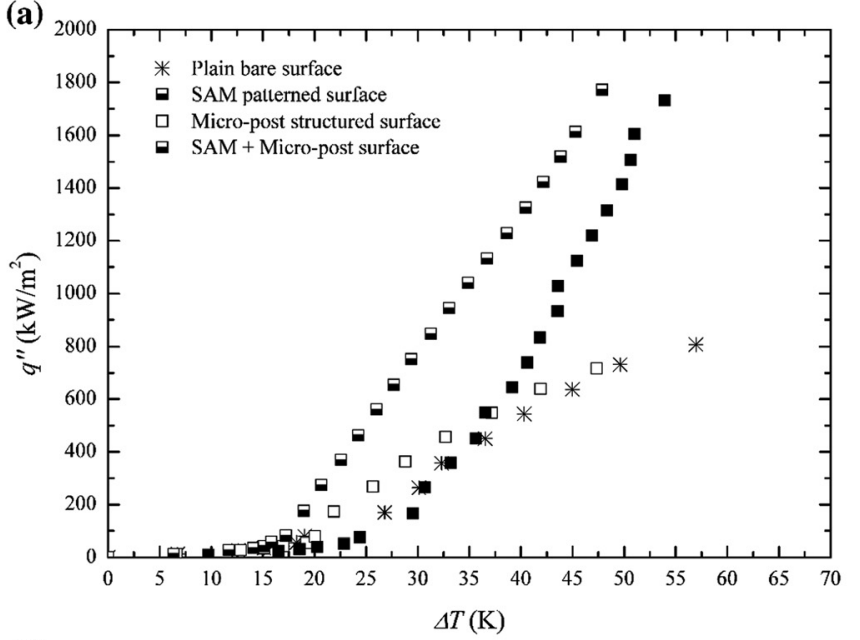

(b)

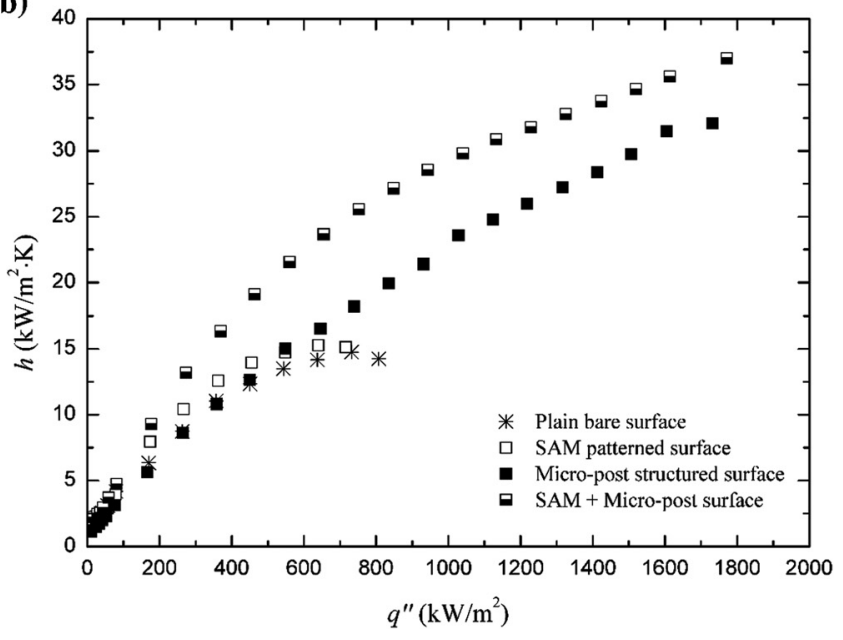

FIG. 2. (a) Boiling curves and (b) boiling heat transfer coefficients on differently modified surfaces.

to form a bubble nucleus. Without the presence of trapped vapors, bubble nucleation for water is predicted at very high temperatures of around $T \sim 300^{\circ} \mathrm{C}$ by classic nucleation theories; ${ }^{22}$ however, the required temperature for water nucleation was found to be $\sim 110^{\circ} \mathrm{C}$. Recently, Jo et al. ${ }^{18}$ postulated a nucleation model that supports this observation of heterogeneous nucleation at reduced superheat on a smooth surface free from trapped vapor. The model supposes that the induced potential gradient within the thermal boundary layer adjacent to the heating surface accelerates the kinetics of a superheated liquid, based on theoretical calculation. Consequently, when thermal equilibrium of the instantly induced bubble nucleus by a high evaporation rate is achieved, a bubble can be nucleated on an ideally smooth or nanostructured surface without trapped vapors. This potential gradient nucleation model predicts a trend of increasing superheat for bubble nucleation with decreasing contact angle. A surface with a lower contact angle has a larger bubble height, requiring higher superheat to satisfy the thermal equilibrium condition for the top of the nucleated bubble. This model predicts that the SAM patterned surface would induce bubble nucleation at a reduced superheat compared to the plain heating surface, due to differences in contact angle on the two surfaces. According to the potential gradient nucleation model, thermal equilibrium is required for the critical state, which has a maximum energy barrier for nucleation. When nucleation superheat increased from 5 to $20^{\circ} \mathrm{C}$, the radius of the critical nucleus decreased from 5.95 to $1.13 \mu \mathrm{m}$-smaller than the SAM pattern. This indicates that the size of the wetting pattern was sufficient to induce bubble nucleation. According to this model, the superheat required on the SAM pattern is $78 \%$ of that needed using the plain surface when the thickness of thermal boundary layer is $100 \mu \mathrm{m}$.

Wettability effects could also affect bubble generation frequency, by reducing the waiting time between subsequent bubble generation events (Fig. 3 and supplementary material $^{20}$ ). The bubble frequency is determined by the growth time of a nucleated bubble and the waiting time between previous bubble departure and next bubble generation. In particular, the waiting time is related with the re-growth of the thermal boundary layer after bubble departure because bubble could be re-nucleated when the superheated water temperature surrounding the bubble nucleus is higher than the required temperature for bubble nucleation. ${ }^{5,8,23}$ Importantly, the nucleus domain is depending on the surface wettability. Therefore, since a bubble nucleated on a surface with higher contact angle has a lower bubble height, the surface can be rapidly re-nucleated by the growing thermal boundary layer from the heating surface. On the SAM patterned surface, the bubble frequency was $60.7 \mathrm{~Hz}$ at $q^{\prime \prime}=80 \mathrm{~kW} / \mathrm{m}^{2}-1.7$-fold larger than on the plain surface $(36.3 \mathrm{~Hz})$. The frequency of bubble generation on the combined SAM pattern/micro-post surface $(87.3 \mathrm{~Hz})$ was also 1.7-fold higher compared with the micro-post only surface $(50.4 \mathrm{~Hz})$. Hence, bubble nucleation is accelerated by the SAM pattern. This supports BHT enhancement on the SAM patterned surface from low heat flux to $\mathrm{CHF}$.

When a bubble expands on the heating surface, the triple line of the bubble base interface should pass through the heating surface; however, the surface heterogeneity interrupts bubble expansion, ${ }^{24-26}$ as shown in Figs. 1(d) and 1(e). At the wetting boundary of the SAM patterns, the heterogeneity of heating surface wettability causes pinning and change in contact angle. However, due to the small contact

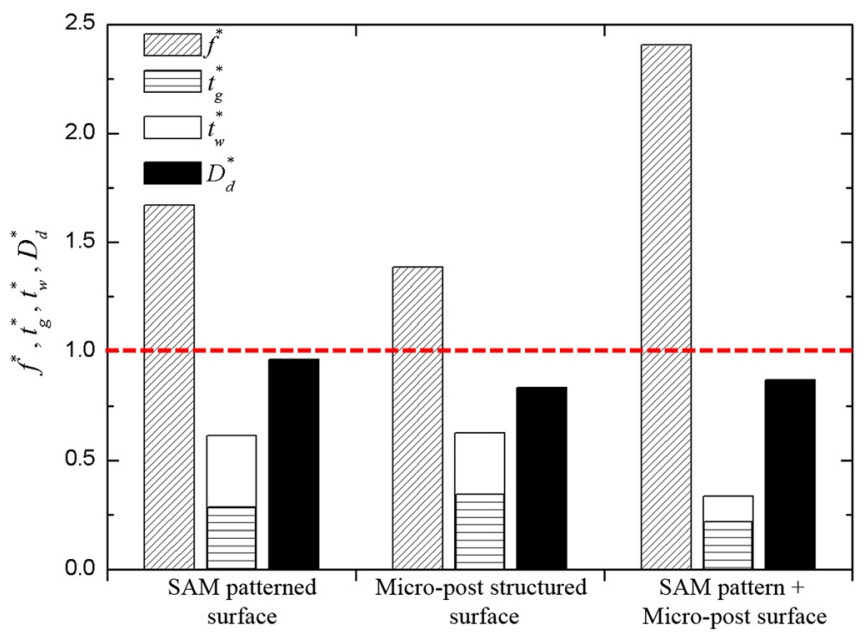

FIG. 3. Reduced frequency $\left(f^{*}\right)$, growth time $\left(t^{*}\right)$, wait time $\left(t^{*}{ }_{\mathrm{w}}\right)$, and departure diameter $\left(D^{*}\right.$ d $)$ of nucleated bubble at $q^{\prime \prime}=79 \mathrm{~kW} / \mathrm{m}^{2}$. The reduction of these parameters was against values measured on the plain surface. 
angle difference between SAM patterns and bare surface, the effect of wettability heterogeneity would not be significant in this study. Meanwhile, the towering pillar geometry of the structured surface would interrupt bubble expansion and the resistance provided by surface structures to bubble dynamics increases as surface roughness increases. The surface roughness is quantitatively defined as the ratio of the total area to the projection. ${ }^{14}$ The surface roughness of the structure surface and the SAM + structure surface were 1.4. Due to resistance on the micro-post structured surface, the base diameter distribution of bubbles on the microstructured surface was smaller than for plain and SAM patterned surfaces, as shown in Fig. 3. This reduced bubble size induced faster bubble generation frequencies on the structured surface. In this study, the departure bubble size $\left(D_{\mathrm{d}}\right)$-frequency $(f)$ relation, affected by the surface structure, can be described as

$$
\text { Constant }=f \cdot D_{\mathrm{d}}^{2} .
$$

As a consequence of the reduced departure diameter, the generation frequency for the structured surface was 1.4-fold higher than for the plain surface. The departure bubble sizefrequency difference between the SAM + structure surface and the SAM-only patterned surface also followed Eq. (1).

The effect of micro-structures on BHT became more significant at high heat flux. As the heat flux increases, the number of activated bubbles increases until it reaches the maximum limit on the heating surface. On the plain surface, when the activated bubble covered the whole heating surface, vapor coalesced, and formed a vapor mushroom on the heating surface. At that point, the interface between the vapor mushroom and the plain heating surface expanded without resistance. For the plain surface, with a heat flux over $\sim 360 \mathrm{~kW} / \mathrm{m}^{2}$, the nucleated bubble covered most of the heating surface, and a vapor mushroom was generated by the coalescence of the nucleated bubble (Fig. 4(a)). At $q^{\prime \prime}>360 \mathrm{~kW} / \mathrm{m}^{2}$, the whole heating surface was covered by the vapor mushroom. On the structured surface, however, there was still space for more bubble activation at the same heat flux. The micro-posts resist the expansion of the vapor mushroom and, as a consequence, a broken interface was observed on the structured surface (Fig. 4(b)). Although the vapor mushroom covered the whole heating surface, the induced liquid path between the broken interfaces resulted in greater liquid supply to the heating surface and maintained high heat-transfer capacity on the structured surface.

This can be confirmed by the instantaneous change in heat flux with respect to instantaneous changes in superheat (d $q^{\prime \prime} / \mathrm{d} \Delta T$ ), equivalent to the slope of boiling curve at each point, and describing the quantity of heat transfer at an instant of time. As shown in Fig. $4, \mathrm{~d} q^{\prime \prime} / \mathrm{d} \Delta T$ on the plain surface increased until $q^{\prime \prime}=360 \mathrm{~kW} / \mathrm{m}^{2}$, with a corresponding increase in activated bubble number. $q^{\prime \prime}$ then deteriorated after vapor mushroom generation. For the structured surface, however, $\mathrm{d} q^{\prime \prime} / \mathrm{d} \Delta T$ increased even past $q^{\prime \prime}=360 \mathrm{~kW} / \mathrm{m}^{2}$ and maintained the maximum state until after CHF. Interestingly, even at saturation of activated bubble number density, the micro-post structured surface transferred heat continuously at the maximum state. This is attributed to the broken interface on the micro-post structured surface.

On the SAM + structure surface, a chemical wetting and mechanical structure pattern hence appears to contribute to improvement of BHT. Bubbles were nucleated on the SAM pattern, located on the heads of micro pillars, and the expansion of the nucleated bubble was disturbed by the micro-post structures. Through these two effects, $\mathrm{d} q^{\prime \prime} / \mathrm{d} \Delta T$ reached a maximum state at comparatively low heat flux. Under high heat flux, furthermore, the vapor path from the SAM patterned micro-post head and the liquid path between

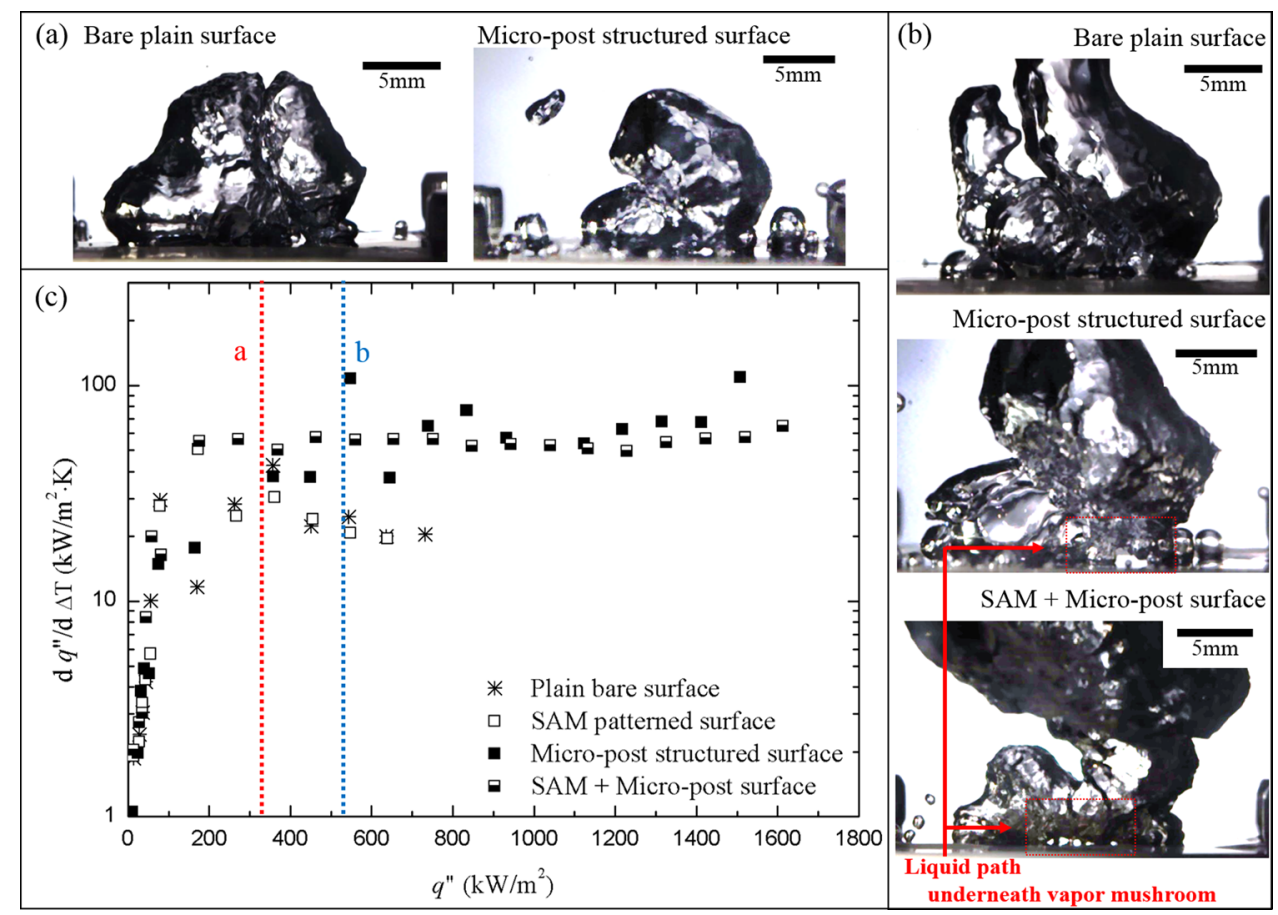

FIG. 4. (a) Visualization images of the vapor mushroom on heating surfaces at $q^{\prime \prime}=360 \mathrm{~kW} / \mathrm{m}^{2}$, (b) at $q^{\prime \prime}=540 \mathrm{~kW} / \mathrm{m}^{2}$, and (c) the instantaneous change in heat flux with respect to instantaneous change in superheat. The instantaneous change was evaluated until the CHF point was almost reached. 
micro-post pillars could be separated, providing stable liquid supply pathways underneath the generated vapor mushroom (Fig. 4(b)). Consequently, the combined surface maintained enhanced BHT and CHF.

The results demonstrated that hydrophilic SAM patterns and micro-post structures spatially fabricated could improve boiling performance by influencing bubble nucleation and nucleated bubble dynamics. In particular, the combined surface, with SAM patterns on the head of micro-post structures, has significant advantages. Bubbles were induced on the head of the micro-posts due to the SAM pattern even for wettability patterns with a contact angle $<90^{\circ}$, and their growth was influenced by both the SAM pattern and micropost structures. Consequently, by separating the vapor path on the SAM patterns and the liquid path between micro-post structures, high BHT was maintained without CHF deterioration. This approach will improve understanding of the effect of spatially variable wetting and structure surface characteristics in two-phase flow systems and could contribute to development of advanced boiling heat transfer systems.

This work was supported by the National Research Foundation of Korea (NRF) grant funded by the Korea government (MSIP) (NRF-2013M2A8A1040987).

${ }^{1}$ S. G. Liter and M. Kaviany, Int. J. Heat Mass Transfer 44(22), 4287-4311 (2001).

${ }^{2}$ C. Li, Z. Wang, P. I. Wang, Y. Peles, N. Koratkar, and G. Peterson, Small 4(8), 1084-1088 (2008).

${ }^{3}$ R. Chen, M.-C. Lu, V. Srinivasan, Z. Wang, H. H. Cho, and A. Majumdar, Nano Lett. 9(2), 548-553 (2009).
${ }^{4}$ H. J. Jo, S. Kim, H. Kim, J. Kim, and M. H. Kim, Nanoscale Res. Lett. 7(1), 242.1-9 (2012).

${ }^{5}$ Y. Hsu, J. Heat Transfer 84(3), 207-213 (1962).

${ }^{6} \mathrm{~S}$. Yang and R. Kim, Int. J. Heat Mass Transfer 31(6), 1127-1135 (1988).

${ }^{7}$ H. J. Jo, H. S. Ahn, S. Kang, and M. H. Kim, Int. J. Heat Mass Transfer 54(25), 5643-5652 (2011).

${ }^{8}$ H. Chi-Yeh and P. Griffith, Int. J. Heat Mass Transfer 8(6), 887-904 (1965).

${ }^{9}$ H. T. Phan, N. Caney, P. Marty, S. Colasson, and J. Gavillet, Int. J. Heat Mass Transfer 52(23), 5459-5471 (2009).

${ }^{10}$ R. Benjamin and A. Balakrishnan, Exp. Therm. Fluid Sci. 15(1), 32-42 (1997).

${ }^{11}$ B. Mikic and W. Rohsenow, J. Heat Transfer 91(2), 245-250 (1969).

${ }^{12}$ S. G. Kandlikar, J. Heat Transfer 123(6), 1071-1079 (2001).

${ }^{13}$ H. S. Ahn, H. J. Jo, S. H. Kang, and M. H. Kim, Appl. Phys. Lett. 98(7), 071908 (2011).

${ }^{14}$ K.-H. Chu, R. Enright, and E. N. Wang, Appl. Phys. Lett. 100(24), 241603 (2012).

${ }^{15}$ Y. Nam and Y. S. Ju, Appl. Phys. Lett. 93(10), 103115 (2008).

${ }^{16}$ A. R. Betz, J. Xu, H. Qiu, and D. Attinger, Appl. Phys. Lett. 97(14), 141909 (2010).

${ }^{17}$ Y. Takata, S. Hidaka, and M. Kohno, Int. J. Air-Cond. Refrig. 20(01), 1150003 (2012).

${ }^{18}$ H. J. Jo, S. H. Kim, H. S. Park, and M. H. Kim, Int. J. Multiphase Flow 62, 101-109 (2014).

${ }^{19}$ A. R. Betz, J. Jenkins, C.-J. Kim, and D. Attinger, Int. J. Heat Mass Transfer 57(2), 733-741 (2013).

${ }^{20}$ See supplementary material at http://dx.doi.org/10.1063/1.4919916 for the fabrication processes, experimental procedure and facility, bubble characteristics, and comparison of experimental results.

${ }^{21}$ S. Bankoff, AIChE J. 4(1), 24-26 (1958).

${ }^{22}$ M. Blander and J. L. Katz, AIChE J. 21(5), 833-848 (1975).

${ }^{23}$ H. J. Jo, M. Kaviany, S. H. Kim, and M. H. Kim, Int. J. Heat Mass Transfer 71, 149-157 (2014).

${ }^{24}$ J. Oliver, C. Huh, and S. Mason, J. Colloid Interface Sci. 59(3), 568-581 (1977).

${ }^{25}$ C. Extrand and A. Gent, J. Colloid Interface Sci. 138(2), 431-442 (1990).

${ }^{26} \mathrm{C}$. Extrand, Langmuir 23(4), 1867-1871 (2007). 